\title{
Associação entre ombro congelado e tireopatias: Reforçando as evidências*
}

\section{Association between Frozen Shoulder and Thyroid Diseases: Strengthening the Evidences}

\author{
Carina Cohen $^{1}$ Simone Tortato ${ }^{1}$ Otavio Bento Souza Silva ${ }^{1}$ Mariana Ferreira Leal ${ }^{1}$ \\ Benno Ejnisman ${ }^{1}$ Flavio Faloppa ${ }^{1}$
}

1 Departamento de Ortopedia e Traumatologia, Escola Paulista de Medicina, Universidade Federal de São Paulo, São Paulo, SP, Brasil

Rev Bras Ortop 2020;55(4):483-489.

\begin{abstract}
Endereço para correspondência Carina Cohen, Departamento de Ortopedia e Traumatologia, Escola Paulista de Medicina, Universidade Federal de São Paulo, Centro de Traumatologia do Esporte, Rua Estado de Israel, 636, São Paulo, SP, Brasil (e-mail: cacohen18@hotmail.com).
\end{abstract}

\section{Resumo}

Palavras-chave

- ombro congelado

- capsulite adesiva

- doenças da tireóide

- hipotireoidismo
Objetivo Verificar a asssociação entre tireopatias e ombro congelado primário, comparando com grupo controle e com grupo de pacientes com lesão no manguito rotador.

Métodos Foram avaliados 166 pacientes com diagnóstico de ombro congelado primário com tratamento em andamento ou já tratados. Este grupo foi comparado com 129 pacientes com diagnóstico de lesão de manguito rotador e com um terceiro grupo controle formado por 251 indivíduos sem acometimento dos ombros. Todos os participantes responderam questionário sobre as seguintes variáveis: idade, gênero, índice de massa corpórea (IMC), profissão, atividade física, presença de tireopatia e de outras comorbidades, hábito tabagista e etilismo.

Resultados Quando comparamos o grupo de ombro congelado com os grupos controle e lesão de manguito rotador, percebemos que existe uma associação específica entre presença de doenças da tireoide (tireoidite, hipotireoidismo, hipertireoidismo, nódulos e câncer) e ombro congelado. Através do cálculo do risco relativo, é possível afirmar que um indivíduo com tireopatia tem probabilidade 2.69 maior de desenvolver ombro congelado. Também houve associação com gênero, já que as mulheres com ombro congelado elevam significativamente esse risco.

Conclusão Os distúrbios da tireoide, especialmente o hipotireoidismo e a presença de nódulos tireoidianos benignos, são fatores de risco significativamente associados ao ombro congelado, aumentando as chances em 2,69 vezes de desenvolver a doença. Este é o primeiro estudo que utiliza, além do grupo controle, um segundo grupo com lesões do manguito rotador, de modo que foi demonstrada uma associação específica de distúrbios da tireoide e ombro congelado.

\footnotetext{
Trabalho desenvolvido na Universidade Federal de São Paulo, Escola Paulista de Medicina, Departamento de Ortopedia e Traumatologia, Centro de Traumatologia do Esporte, São Paulo, SP, Brasil.
}

recebido

04 de Novembro de 2018 aceito

26 de Fevereiro de 2019
DOI https://doi.org/

10.1055/s-0039-3402476. ISSN 0102-3616.
Copyright $\odot 2020$ by Sociedade Brasileira License terms de Ortopedia e Traumatologia. Published by Thieme Revinter Publicações Ltda, Rio de Janeiro, Brazil 


\begin{abstract}
Objective To clarify the association of thyroid disorders and primary frozen shoulder by comparing this group with controls without shoulder disease and with patients with rotator cuff tears.

Methods We evaluated 166 patients who presented frozen shoulder with treatment in progress or already treated, which were compared with 129 patients with diagnosis of rotator cuff tears and 251 control subjects. All of the participants answered the questionnaire on the following variables: age, gender, body mass index (BMI), occupation, physical activity, presence of thyroid disorders and other comorbidities, smoking and use of alcohol.

Results When comparing the frozen shoulder group with the control and rotator cuff groups, there is a specific association between the presence of thyroid disorders and frozen shoulder. By calculating relative risk, it is possible to state that an individual with thyropathy has 2.69 more chance of developing frozen shoulder. Also, there was an association with gender, since women with frozen shoulder exceeded significantly the risk.

Conclusions Thyroid disorders, especially hypothyroidism and the presence of benign thyroid nodules, are risk factors significantly associated with frozen shoulder, rising the chances to 2.69 times of developing frozen shoulder.

- frozen shoulder This is the first study that uses, in addition to the control group, a second group with

- adhesive capsulitis

- thyroid diseases

- hypothyroidism rotator cuff tears, so it was shown that there is a specific association of thyroid disorders and frozen shoulder, but not with shoulder disorders in general.
\end{abstract}

\section{Introdução}

O ombro congelado é uma condição clínica que acomete a articulação glenoumeral, causando dor e limitação da amplitude de movimento. Por ter um curso insidioso, é difícil definir sua incidência exata, pois muitos pacientes não procuram atendimento médico. Em geral, afeta de 2 a 5\% da população, com uma leve predileção pelo sexo feminino. ${ }^{1}$ 0 pico de incidência ocorre entre 40 a 60 anos e história familiar positiva está presente em até 9,5\% dos casos. ${ }^{2}$

Essa condição clínica foi descrita em 1896 por Duplay ${ }^{3}$ como uma periartrite escapuloumeral. Já Codman, ${ }^{4}$ em 1934 , criou o termo "ombro congelado" para denominar os casos de rigidez dolorosa do ombro, de longa duração e de início muitas vezes relacionado com períodos de imobilização ou de desuso da articulação. Desde então, várias definições e classificações foram propostas. O consenso da American Shoulder and Elbow Surgeons a define como "uma condição de etiologia incerta, caracterizada por significante restrição de movimento ativo e passivo do ombro, que ocorre na ausência de uma desordem intrínseca conhecida do ombro." 5 O consenso mais recente publicado pelo comitê de membro superior da International Society of Arthroscopy Knee and Orthopedic Surgery (ISAKOS, na sigla em inglês) desencoraja o uso do termo amplamente utilizado "capsulite adesiva," e define ombro rígido como um termo global que abrange qualquer restrição de amplitude de movimento do ombro, sendo classificado, de acordo com a etiologia, em ombro congelado (ou primário idiopático) e secundário. ${ }^{6}$ Desta forma, o termo "ombro congelado" seria usado exclusiva- mente para descrever aqueles casos de ombro rígido de causa desconhecida. Por outro lado, o termo "ombro rígido secundário" fica restrito aos casos secundários a trauma, cirurgia ou doença específica do próprio ombro (lesão do manguito rotador, fraturas e tendinite calcárea $)^{6}$

Existem vários fatores, embora o mecanismo de atuação deles seja desconhecido, que podem predispor a um ombro rígido, como diabetes mellitus (DM), tireopatias (tireoidite, hipotireoidismo, hipertireoidismo, nódulos e câncer), neoplasias, doenças cardíacas, pulmonares e neurológicas, contratura de Dupuytren, fatores emocionais, uso de determinados medicamentos e imobilização prévia. $^{6-10}$

Assim como outras doenças já mencionadas, as tireopatias são citadas em vários estudos como um dos fatores associados ao ombro congelado e a outros transtornos musculoesqueléticos, porém ainda sem uma compreensão adequada. $8,11-13$

O termo tireopatia agrupa uma série de doenças da tireoide, sendo o hipotireoidismo, caracterizado por uma deficiência de produção do hormônio pela glândula tiroide, a mais comumente encontrada na população em geral. Embora a prevalência do hipertireoidismo tenha sido relatada em $1,5 \%$, o hipotireoidismo é quase 10 vezes mais frequente. A sua prevalência é variável de acordo com estudos (6-20\%) dependendo da idade, gênero, raça, iodossuficiência da população estudada, bem como do nível de referência do hormônio tireoestimulante (TSH). ${ }^{14-16}$

Alguns autores sugerem uma etiologia autoimune na relação entre a tireoidite de Hashimoto, que é a causa mais comum de hipotireoidismo na população em geral, e o ombro congelado. $9,11,12,17$ 
Assim, o presente estudo teve como objetivo verificar a associação entre tireopatias e ombro congelado, comparando este com grupo controle de indivíduos sem acometimento dos ombros e com um grupo de pacientes com lesão no manguito rotador, considerando fatores como idade de acometimento, gênero, bilateralidade e tempo de lesão.

\section{Material e métodos}

\section{Casuística}

Entre junho de 2012 a maio de 2016, no ambulatório de ombro e cotovelo da nossa instituição, foram recrutados pacientes com diagnóstico de ombro congelado e indivíduos com lesão no manguito rotador. No grupo 1, foram incluídos 166 pacientes com diagnóstico de ombro congelado primário com tratamento em andamento ou já tratados. O diagnóstico foi realizado pelo exame clínico (pacientes com dor a mobilização ativa e passiva do ombro e restrição global do movimento em comparação ao lado contralateral) e confirmado por ressonância magnética (RM) em todos os casos. A RM foi utilizada principalmente para descartar diagnósticos diferenciais. Dessa forma, foram excluídos os pacientes que apresentaram lesões de manguito rotador, tendinite calcárea, luxação do ombro, traumas, fraturas ou cirurgia prévia por se tratarem de casos de ombro rígido secundário, e não ombro congelado.

O grupo 2 foi formado por 129 pacientes com diagnóstico clínico e por RM de lesão de manguito rotador sem evidências clínicas e radiológicas de ombro congelado, pareados por gênero e idade com o grupo 1 .

O grupo 3 foi o grupo controle, composto por 251 indivíduos atendidos no serviço de ortopedia, sem qualquer queixa, trauma ou diagnóstico de doença ortopédica que envolvesse a articulação do ombro.

Todos os participantes do estudo passaram por uma avaliação clínica e responderam, conduzidos por entrevista de um único médico, o mesmo questionário sobre as seguintes variáveis: idade, gênero, peso/altura para cálculo de índice de massa corpórea (IMC), profissão, atividade física, presença de tireopatia diagnosticada por médico e de outras comorbidades (DM, hipertensão arterial sistêmica [HAS], cardiopatias, distúrbios psiquiátricos), hábito tabagista e etilismo. Para os pacientes com ombro congelado, foi também avaliado o lado e dominância do braço acometido, bem como o tempo de duração da doença.

Todos os participantes do estudo assinaram o termo de consentimento livre esclarecido, cujo modelo foi aprovado pelo Comitê de Ética em Pesquisa da nossa instituição (parecer 51436).

\section{Análise estatística}

A análise estatística foi realizada utilizando-se o programa PASW Statistics for Windows, versão 18.0 (SPSS Inc., Chicago, IL, EUA). O teste Kolmogorov-Smirnov foi utilizado para verificar a distribuição das variáveis contínuas. Quando as variáveis não seguiram uma distribuição normal, optamos por apresentar os resultados em mediana e desvio interquartílico. Realizamos três análises comparando os seguintes grupos: a) ombro congelado (grupo 1) e controle (grupo
3); b) manguito rotador (grupo 2) e controle (grupo 3) e c) ombro congelado (grupo 1) e manguito rotador (grupo 2). Para tanto, realizamos uma regressão logística univariada considerando o grupo 1 ou grupo 2 como variável dependente e cada variável clínica ou demográfica como variável independente. Posteriormente, as variáveis significativamente associadas com cada uma das doenças foram incluídas em uma regressão logística multivariada. Essas análises foram corrigidas para três comparações. Diferenças estatísticas significativas foram consideradas quando $p<0,05$.

\section{Descrição da Amostra}

O grupo 1 foi formado por $60 \%$ pacientes do sexo feminino com idade de acometimento variando de 30 a 74 anos (Mediana \pm desvio interquatílico [DI]: $51 \pm 11$, - Tabela 1). O acometimento bilateral foi observado em $20 \%$ dos pacientes. Dentre os casos unilaterais, o lado dominante foi afetado em 54\%. Do total de pacientes, 57\% encontravam-se em fase ativa da doença. Para os demais pacientes, considerados tratados, o tempo de duração da doença variou de 1 a 60 meses (mediana \pm DI: $8 \pm 6$ ). Além disso, $34 \%$ dos pacientes referiram diagnóstico médico, confirmado previamente por exames de sangue ou de imagem, de alguma tireopatia (-Tabela 1). Outras afecções foram relatadas, como doenças psiquiátricas (33\%), dislipidemia (26\%), hipertensão (22\%), litíase renal (13\%), diabetes (11\%), neoplasias malignas (10\%), fibromialgia ( $8 \%$ ) e hiperuricemia (2\%). Etilismo foi relatado em $5 \%$ e tabagismo em $37 \%$. Em relação ao índice de massa corpórea (IMC), 50\% dos pacientes apresentaram sobrepeso ou obesidade. Adicionalmente, $14 \%$ relataram desempenhar atividade profissional manual e 55\% relataram a prática de atividade física (-Tabela $\mathbf{1}$ ).

O grupo 2 foi formado por $59 \%$ de pacientes do sexo feminino e com idade de acometimento variando de 28 a 59 anos (mediana \pm DI: $52 \pm 8$, - Tabela 2). Além disso, 5\% dos pacientes referiram diagnóstico médico prévio de alguma tireopatia (-Tabela 2). Outras afecções foram relatadas, como hipertensão (52\%), dislipidemia (21\%), diabetes (7\%), fibromialgia (7\%), doenças psiquiátricas (6\%), litíase renal (3\%), neoplasias malignas (1\%) e hiperuricemia (1\%). Etilismo foi relatado em 16\% e o tabagismo em 36\%. Em relação ao IMC, $62 \%$ dos pacientes apresentaram sobrepeso ou obesidade. Adicionalmente, $43 \%$ relataram desempenhar atividade profissional manual e $35 \%$ relataram a prática de atividade física (-Tabela 2 ).

O grupo 3 foi formado por $60 \%$ de indivíduos do sexo feminino com idade variando de 30 a 75 anos (mediana \pm DI: $50 \pm 10$, - Tabelas 1 e 2). Do total desses indivíduos, $14 \%$ referiram diagnóstico médico prévio de alguma tireopatia (-Tabelas 1 e 2). Outras afecções foram relatadas, como HAS (37\%), DM (18\%), dislipidemia (17\%), neoplasias malignas $(8 \%)$, doenças psiquiátricas (3\%), fibromialgia diagnosticada (1\%) e litíase renal (1\%). Etilismo foi relatado em $18 \%$ e o tabagismo em 39\%. Em relação ao IMC, 60\% dos indivíduos apresentaram sobrepeso ou obesidade. Adicionalmente, 50\% relataram desempenhar atividade profissional manual e $41 \%$ relataram a prática de atividade física (-Tabelas 1 e 2 ). 
Tabela 1 Análise de regressão logística univariada com variáveis associadas ao desenvolvimento do ombro congelado (grupo 1) em relação aos indivíduos controles (grupo 3)

\begin{tabular}{|c|c|c|c|c|c|}
\hline Variáveis clínicas e demográficas & Grupo 1 & Grupo 3 & OR & IC 95\% & valor-p \\
\hline Idade (Mediana \pm DI) & $51 \pm 11$ & $50 \pm 10$ & 1,01 & $0,99-1,04$ & 0,26 \\
\hline Gênero feminino [N(\%)] & $100(60 \%)$ & $151(60 \%)$ & 1,00 & $0,67-1,50$ & 0,99 \\
\hline Sobrepeso ou obesidade ${ }^{\mathrm{a}}[\mathrm{N}(\%)]$ & 83 (50\%) & $152(60 \%)$ & 0,65 & $0,44-0,97$ & 0,03 \\
\hline Profissão manual [N(\%)] & $23(14 \%)$ & $126(50 \%)$ & 0,16 & $0,10-0,26$ & $<0,01^{*}$ \\
\hline Atividade física [N(\%)] & $92(55 \%)$ & $103(41 \%)$ & 1,79 & $1,20-2,70$ & $<0,01^{*}$ \\
\hline Tireoidopatia [N(\%)] & $56(34 \%)$ & $35(14 \%)$ & 3,14 & $1,94-5,08$ & $<0,01^{*}$ \\
\hline Tireoidite $[\mathrm{N}(\%)]$ & $6(4 \%)$ & $4(2 \%)$ & 2,32 & $0,64-8,33$ & 0,20 \\
\hline Hipotiroidismo [N(\%)] & $29(17 \%)$ & $21(8 \%)$ & 2,32 & $1,27-4,23$ & $<0,01^{*}$ \\
\hline Nódulos benignos [N(\%)] & $16(10 \%)$ & $6(2 \%)$ & 4,36 & $1,67-11,38$ & $<0,01^{*}$ \\
\hline Câncer [N(\%)] & $5(3 \%)$ & $4(2 \%)$ & 1,92 & $0,51-7,25$ & 0,34 \\
\hline Diabetes [N(\%)] & $19(11 \%)$ & $46(18 \%)$ & 0,58 & $0,32-1,02$ & 0,06 \\
\hline Dislipidemia [N(\%)] & $43(26 \%)$ & $43(17 \%)$ & 1,69 & $1,05-2,73$ & 0,03 \\
\hline Litíase renal $[\mathrm{N}(\%)]$ & $22(13 \%)$ & $2(1 \%)$ & 19,02 & $4,41-82,07$ & $<0,01^{*}$ \\
\hline Fibromialgia [N(\%)] & $13(8 \%)$ & $2(1 \%)$ & 10,58 & $2,36-47,52$ & $<0,01^{*}$ \\
\hline Hipertensão [N(\%)] & $37(22 \%)$ & $92(37 \%)$ & 0,50 & $0,32-0,78$ & $<0,01^{*}$ \\
\hline Hiperurecemia [N(\%)] & $3(2 \%)$ & $0(0 \%)$ & 2,49 & $<0,01-<0,01$ & 0,99 \\
\hline Neoplasias malignas [N(\%)] & $16(10 \%)$ & $20(8 \%)$ & 1,23 & $0,62-2,45$ & 0,55 \\
\hline Doenças psiquiátricas [N(\%)] & $54(33 \%)$ & $8(3 \%)$ & 14,65 & $6,74-31,81$ & $<0,01^{*}$ \\
\hline Medicação de uso continuo [N(\%)] & $112(66 \%)$ & $179(71 \%)$ & 0,83 & $0,55-1,28$ & 0,40 \\
\hline Etilismo $^{\mathrm{b}}[\mathrm{N}(\%)]$ & $9(5 \%)$ & $45(18 \%)$ & 0,26 & $0,13-0,55$ & $<0,01^{*}$ \\
\hline Tabagismo $^{\mathrm{C}}[\mathrm{N}(\%)]$ & $62(37 \%)$ & 98 (39\%) & 0,93 & $0,62-1,39$ & 0,70 \\
\hline
\end{tabular}

Abreviações: DI, desvio interquartílico; IC, intervalo de confiança; N, número de pacientes; OR (odds ratio), razão de chances de um evento ocorrer. aIMC $\geq 25$.

${ }^{\mathrm{b}}$ Etilistas e ex-etilistas.

${ }^{\mathrm{C}}$ Tabagistas e ex-tabagistas.

${ }^{*} p<0,02$.

\section{Resultados}

A análise de regressão logística univariada demonstrou diferentes variáveis associadas ao desenvolvimento de ombro congelado e da lesão de manguito rotador, em relação aos indivíduos controle (-Tabelas 1, 2 e 3). Quando essas variáveis foram inseridas no modelo de regressão logística multivariada, houve associação entre o desenvolvimento de ombro congelado e tireopatias (tireoidites, hipotireoidismo, hipertireoidismo, nódulos e câncer de tireoide), litíase renal e doença psiquiátrica (depressão, distúrbios do sono e de ansiedade, transtornos comportamentais diagnosticados e tratados por médico), em relação ao grupo 3. Em relação aos indivíduos com lesão de manguito rotador, tireopatia, neoplasias malignas, doenças psiquiátricas, hipertensão e etilismo foram associados com o desenvolvimento de ombro congelado.

Em relação à tireopatia (incluindo todas as categorias) e comparando o grupo 1 com os grupos 2 e 3 , é possível afirmar que existe uma associação entre o desenvolvimento de algum tipo de tireopatia e ombro congelado ( - Tabelas 1 e 2), mesmo corrigindo-se para diferentes covariáveis. Essa associação não foi observada nos pacientes com lesão de manguito rotador, comparando-os com o grupo controle (-Tabela 3). Portanto, o desenvolvimento de algum tipo de tireopatia parece ser específico nos casos de ombro congelado. Nesse grupo, os tipos de alterações na tireoide relatados foram hipotireoidismo (17\%), nódulos benignos (10\%), tireoidite (4\%) e câncer de tireoide (3\%) (-Tabela 1). A presença de hipotireoidismo e nódulos benignos foi associada ao desenvolvimento do ombro congelado em relação aos indivíduos controle ( - Tabela 1 ) e com lesão no manguito rotador (-Tabela 2).

Ainda no grupo 1, a grande maioria dos pacientes portadores de tireopatias eram mulheres (84\%), sendo que a análise de regressão logística mostrou uma associação significativa entre o acometimento da tireoide e gênero, de forma que mulheres com ombro congelado têm maior risco de apresentar a doença (-Tabela 4). A idade dos indivíduos tireopatas neste grupo variou de 30 a 71 anos (mediana \pm DI: $51 \pm 11$ ) e não diferiu da idade dos indivíduos sem tireopatia (-Tabela 4). 0 ombro congelado foi bilateral em $20 \%$ dos tireopatas e não diferiu dos pacientes sem tireopatia (-Tabela 4). O tempo de lesão dos pacientes tireopatas 
Tabela 2 Análise de regressão logística univariada incluindo variáveis associadas ao desenvolvimento do ombro congelado (grupo 1) em relação aos indivíduos com lesão no manguito rotador (grupo 2)

\begin{tabular}{|l|l|l|l|l|l|}
\hline Variáveis clínicas e demográficas & Grupo 1 & Grupo 2 & OR & IC 95\% & valor-p \\
\hline Idade (Mediana $\pm \mathrm{DI})$ & $51 \pm 11$ & $52 \pm 8$ & 1,00 & $0,97-1,03$ & 0,99 \\
\hline Gênero feminino [N(\%)] & $100(60 \%)$ & $76(59 \%)$ & 1,06 & $0,66-1,69$ & 0,82 \\
\hline Sobrepeso ou obesidadea [N(\%)] & $83(50 \%)$ & $80(62 \%)$ & 0,61 & $0,38-0,98$ & 0,04 \\
\hline Profissão manual [N(\%)] & $23(14 \%)$ & $56(43 \%)$ & 0,21 & $0,12-0,37$ & $<0,01^{*}$ \\
\hline Atividade física [N(\%)] & $92(55 \%)$ & $45(35 \%)$ & 2,32 & $1,45-3,73$ & $<0,01^{*}$ \\
\hline Tireoidopatia [N(\%)] & $56(34 \%)$ & $7(5 \%)$ & 8,87 & $3,88-20,29$ & $<0,01^{*}$ \\
\hline Tireoidite [N(\%)] & $6(4 \%)$ & $0(0 \%)$ & 13,02 & $<0,01-<0,01$ & 0,99 \\
\hline Hipotiroidismo [N(\%)] & $29(17 \%)$ & $5(4 \%)$ & 5,25 & $1,97-13,98$ & $<0,01^{*}$ \\
\hline Nódulos benignos [N(\%)] & $16(10 \%)$ & $2(2 \%)$ & 6,77 & $1,53-30,02$ & $0,01^{*}$ \\
\hline Câncer [N(\%)] & $5(3 \%)$ & $0(0 \%)$ & 12,94 & $<0,01-<0,01$ & 0,99 \\
\hline Diabetes [N(\%)] & $19(11 \%)$ & $9(7 \%)$ & 1,72 & $0,75-3,95$ & 0,20 \\
\hline Dislipidemia [N(\%)] & $43(26 \%)$ & $27(21 \%)$ & 1,32 & $0,76-2,29$ & 0,32 \\
\hline Litíase renal [N(\%)] & $22(13 \%)$ & $4(3 \%)$ & 4,77 & $1,60-14,23$ & $<0,01^{*}$ \\
\hline Fibromialgia [N(\%)] & $13(8 \%)$ & $9(7 \%)$ & 1,13 & $0,47-2,74$ & 0,78 \\
\hline Hipertensão [N(\%)] & $37(22 \%)$ & $67(52 \%)$ & 0,27 & $0,16-0,44$ & $<0,01^{*}$ \\
\hline Hiperurecemia [N(\%)] & $3(2 \%)$ & $1(1 \%)$ & 2,36 & $0,24-22,92$ & 0,46 \\
\hline Neoplasias malignas [N(\%)] & $16(10 \%)$ & $1(1 \%)$ & 13,65 & $1,79-104,38$ & $0,01^{*}$ \\
\hline Doenças psiquiátricas [N(\%)] & $54(33 \%)$ & $8(6 \%)$ & 7,29 & $3,32-16,00$ & $<0,01^{*}$ \\
\hline Uso de medicação continua [N(\%)] & $112(66 \%)$ & $81(63 \%)$ & 1,23 & $0,76-1,99$ & 0,40 \\
\hline Etilismob [N(\%)] & $9(5 \%)$ & $21(16 \%)$ & 0,30 & $0,13-0,67$ & $<0,01^{*}$ \\
\hline Tabagismoc [N(\%)] & $62(37 \%)$ & $47(36 \%)$ & 0,99 & $0,61-1,60$ & 0,97 \\
\hline
\end{tabular}

Abreviações: DI, desvio interquartílico; IC, intervalo de confiança; N, número de pacientes; OR (odds ratio), razão de chances de um evento ocorrer. aIMC $\geq 25$.

${ }^{\mathrm{b}}$ Etilistas e ex-etilistas.

${ }^{\mathrm{C}}$ Tabagistas e ex-tabagistas. ${ }^{*} p<0,02$.

variou de 1 a 36 meses (mediana $\pm \mathrm{DI}: 7 \pm 7$ ) e não diferiu daqueles pacientes sem tireopatia (-Tabela 4 ).

\section{Discussão}

Analisando os 3 grupos, percebemos uma prevalência aumentada das doenças da tireoide no grupo 1 (34\%), quando comparada aos demais grupos e mesmo à literatura sobre tireopatias. Segundo Huang et al. ${ }^{13}$ que estudou a prevalência e o risco de desenvolvimento de ombro congelado em pacientes portadores de hipertireoidismo, os indivíduos com hipertiroidismo têm 1,22 vezes o risco de desenvolver a doença em comparação com a população em geral. Em nosso estudo, encontramos apenas dois pacientes com diagnóstico de hipertireoidismo, ambos no grupo 2. Mas, considerando os grupos pareados por sexo e idade (grupos 1 e 3), é possível afirmar que existe uma maior frequência de tireopatias em pacientes com ombro congelado quando comparados ao grupo controle. Calculando o risco relativo, é possível afirmar que um indivíduo com tireopatia tem 2,69 mais chance de desenvolver o ombro congelado. Não foi observada uma associação entre as tireopatias e o grupo 2, sugerindo uma associação direta com ombro congelado, mas não com as lesões do manguito rotador.

Analisando apenas os pacientes com hipotireoidismo, observamos prevalência significativamente aumentada (17\%) no grupo do ombro congelado. No grupo 2, a prevalência foi de $4 \%$, e no grupo 3, de $2 \%$. Dados semelhantes foram encontrados por Schiefer et al. ${ }^{17}$ em estudo caso controle com 93 pacientes com ombro congelado e 151 casos controle, no qual observou prevalência de hipotireoidismo em $27,2 \%$ e $10,7 \%$, respectivamente. Além disso, relatou uma tendência de associação independente entre altos níveis séricos de TSH, bilateralidade e gravidade do ombro congelado.

Em outro estudo, um caso controle realizado na China com 182 casos de ombro congelado, foram identificados $25 \%$ de tireopatas e $24 \%$ de diabéticos, contra $16 \%$ e $9 \%$ respectivamente no grupo controle. $^{18}$

Segundo estudo de Milgrom et al. que analisou dados de 126 ombros congelados de 98 pacientes da cidade de Jerusalém, as tireopatias representam não apenas fator de risco para transtornos musculoesqueléticos em geral, mas também um fator de risco específico para ombro congelado em mulheres. ${ }^{19}$ 
Tabela 3 Análise de regressão logística univariada incluindo variáveis associadas à lesão no manguito rotador (grupo 2) em relação aos indivíduos controles (grupo 3)

\begin{tabular}{|c|c|c|c|c|c|}
\hline Variáveis clínicas e demográficas & Grupo 2 & Grupo 3 & OR & IC 95\% & valor-p \\
\hline Idade (Mediana \pm DI) & $52 \pm 8$ & $50 \pm 10$ & 1,02 & $0,99-1,04$ & 0,29 \\
\hline Gênero feminino [N(\%)] & $76(59 \%)$ & $151(60 \%)$ & 0,95 & $0,62-1,46$ & 0,82 \\
\hline Sobrepeso ou obesidade ${ }^{\mathrm{a}}[\mathrm{N}(\%)]$ & $80(62 \%)$ & $152(60 \%)$ & 1,06 & $0,69-1,65$ & 0,78 \\
\hline Profissão manual [N(\%)] & $56(43 \%)$ & $126(50 \%)$ & 0,76 & $0,50-1,17$ & 0,21 \\
\hline Atividade física [N(\%)] & $45(35 \%)$ & $103(41 \%)$ & 0,77 & $0,50-1,20$ & 0,25 \\
\hline Tireoidopatia [N(\%)] & $7(5 \%)$ & $35(14 \%)$ & 0,35 & $0,15-0,82$ & 0,03 \\
\hline Tireoidite $[\mathrm{N}(\%)]$ & $0(0 \%)$ & $4(2 \%)$ & $<0,01$ & $<0,01-<0,01$ & 0,99 \\
\hline Hipotiroidismo [N(\%)] & $5(4 \%)$ & $21(8 \%)$ & 0,44 & $0,16-1,20$ & 0,11 \\
\hline Nódulos benignos [N(\%)] & $2(2 \%)$ & $6(2 \%)$ & 0,64 & $0,13-3,23$ & 0,59 \\
\hline Câncer [N(\%)] & $0(0 \%)$ & $4(2 \%)$ & $<0,01$ & $<0,01-<0,01$ & 0,99 \\
\hline Diabetes [N(\%)] & $9(7 \%)$ & $46(18 \%)$ & 0,33 & $0,16-0,71$ & $<0,01^{*}$ \\
\hline Dislipidemia [N(\%)] & $27(21 \%)$ & $43(17 \%)$ & 1,28 & $0,75-2,19$ & 0,37 \\
\hline Litíase renal $[\mathrm{N}(\%)]$ & $4(3 \%)$ & $2(1 \%)$ & 3,98 & $0,72-22,05$ & 0,11 \\
\hline Fibromialgia [N(\%)] & $9(7 \%)$ & $2(1 \%)$ & 9,34 & $1,99-43,89$ & $<0,01^{*}$ \\
\hline Hipertensão [N(\%)] & $67(52 \%)$ & $92(37 \%)$ & 1,87 & $1,21-2,87$ & $<0,01^{*}$ \\
\hline Hiperurecemia [N(\%)] & $1(1 \%)$ & $0(0 \%)$ & 3,17 & $<0,01-<0,01$ & 1,00 \\
\hline Neoplasias malignas [N(\%)] & $1(1 \%)$ & $20(8 \%)$ & 0,09 & $0,01-0,68$ & 0,03 \\
\hline Doenças psiquiátricas [N(\%)] & $8(6 \%)$ & $8(3 \%)$ & 2,01 & $0,74-5,48$ & 0,17 \\
\hline Uso de medicação continua [N(\%)] & $81(63 \%)$ & $179(71 \%)$ & 0,68 & $0,43-1,06$ & 0,09 \\
\hline Etilismo $^{\mathrm{b}}[\mathrm{N}(\%)]$ & $21(16 \%)$ & $45(18 \%)$ & 0,89 & $0,50-1,57$ & 0,69 \\
\hline Tabagismo $^{\mathrm{C}}[\mathrm{N}(\%)]$ & $47(36 \%)$ & $98(39 \%)$ & 0,94 & $0,60-1,45$ & 0,76 \\
\hline
\end{tabular}

Abreviações: DI, desvio interquartílico; IC, intervalo de confiança; N, número de pacientes; OR (odds ratio), razão de chances de um evento ocorrer. ${ }^{\mathrm{a}} \mathrm{IMC} \geq 25$.

bEtilistas e ex-etilistas.

${ }^{\mathrm{C}}$ Tabagistas e ex-tabagistas.

${ }^{*} p<0,02$.

Tabela 4 Associação entre tireopatias e variáveis clínicas dos pacientes com ombro congelado

\begin{tabular}{|l|l|l|l|l|l|}
\hline Variáveis clínicas e demográficas & Tireopatas & Não-tireopatas & OR & IC95\% & valor-p \\
\hline Idade de acometimento (Mediana $\pm \mathrm{DI})$ & $51 \pm 11$ & $51 \pm 10$ & 1,02 & $0,97-1,06$ & 0,47 \\
\hline Gênero feminino $[\mathrm{N}(\%)]$ & $47(84 \%)$ & $53(48 \%)$ & 5,62 & $2,51-12,56$ & $<0,01^{*}$ \\
\hline Bilateralidade $[\mathrm{N}(\%)]$ & $11(20 \%)$ & $23(21 \%)$ & 0,93 & $0,41-2,07$ & 0,85 \\
\hline Tempo de lesão em meses (mediana $\pm \mathrm{DI})$ & $7 \pm 7$ & $8 \pm 7$ & 0,99 & $0,93-1,05$ & 0,77 \\
\hline
\end{tabular}

Abreviações: DI, desvio interquartílico; IC, intervalo de confiança; N, número de pacientes; OR (odds ratio), razão de chances de um evento ocorrer. ${ }^{*} p<0,05$.

Cakir et al. avaliou pacientes de uma clínica endocrinológica e constatou que entre pacientes com tireopatia, o ombro congelado estava presente em $10,9 \%$ dos casos, contratura de Dupuytren em $8,8 \%$, síndrome do túnel do carpo em 9,5\%, limitação da amplitude de movimento articular em 4,4\% e dedo em gatilho em $2,9 \%$ dos casos. ${ }^{11}$

Quando analisamos o tempo de lesão, bilateralidade e idade de acometimento, não houve diferença significativa entre os grupos com e sem tireopatia, ou seja, o fato de ter tireopatia parece não influenciar esses fatores. Apesar de Reeves $^{20}$ sugerir que a doença apresente resolução espontâ- nea após 30 meses, Schiefer et al. ${ }^{17}$ encontraram dor residual em $50 \%$ dos pacientes e algum déficit de movimento em $60 \%$.

Apesar de muitos estudos evidenciarem a relação do ombro congelado com o $\mathrm{DM},{ }^{21-26}$ em nosso estudo não foi possível confirmar tal associação. Zreik et al. ${ }^{26} \mathrm{em}$ uma metaanálise de prevalência, identificaram que pacientes diabéticos têm cinco vezes mais chance de desenvolver ombro congelado do que pacientes não diabéticos. Nos pacientes diabéticos, o ombro congelado mostrou-se mais severo e mais resistente ao tratamento, assim como encontrado no presente estudo, quando se observou um curso mais 
arrastado da doença, com tempo maior de tratamento quando comparado ao grupo sem diabetes.

No passado, o DM foi a principal doença associada ao ombro congelado, mas estudos recentes corroboram a associação do ombro congelado com as doenças da tireoide. ${ }^{27}$ Possivelmente, porque o diagnóstico das tireopatias não era valorizado, e atualmente o screening de hormônios tireoidianos faz parte da rotina, o diagnostico é feito com maior frequência e a doença é melhor compreendida.

Nosso estudo apresenta algumas limitações. A primeira delas é com relação aos diferentes estágios de doença dos pacientes com ombro congelado. Apesar de o questionário ser apropriado ao estudo, existem pacientes com diagnóstico recente, pacientes em tratamento e pacientes tratados. Como o objetivo era analisar a associação clínica com a presença de tireopatias, essa heterogeneidade não interfere para os achados; entretanto, enfraquece nossa análise em relação ao curso da doença nesses indivíduos. Outra limitação é a ausência de informações com relação aos níveis dos hormônios tireoidianos, já que foram inclusos como tireopatas aqueles pacientes que relataram diagnóstico médico prévio da doença, porém não foi realizada a reconfirmação diagnóstica laboratorial ou por imagem neste estudo. Dessa forma, casos de hipotireoidismo subclínico não foram incluídos.

Apesar das limitações, nosso estudo é o primeiro que utiliza, além do grupo controle, um segundo grupo com acometimento do ombro por lesão do manguito rotador. Como alguns estudos prévios mostraram uma associação de doenças da tireoide com transtornos musculoesqueléticos, essa escolha foi realizada para determinar a existência de associação específica das tireopatias com ombro congelado.

\section{Conclusão}

Como conclusões do presente estudo, podemos afirmar que as tireopatias, em especial o hipotireoidismo e a presença de nódulos benignos da tireoide, são fatores associados ao ombro congelado. Essa associação não foi observada nos casos de lesão do manguito rotador. Outros fatores, tais como idade de acometimento, gênero, bilateralidade e tempo de lesão não foram influenciados pela presença de tireopatias nos pacientes com ombro congelado.

Conflito de Interesses

Os autores declaram não haver conflito de interesses.

\section{Referências}

1 Hsu JE, Anakwenze OA, Warrender WJ, Abboud JA. Current review of adhesive capsulitis. J Shoulder Elbow Surg 2011;20(03):502-514

2 Cohen C, Ejnisman B. Epidemiology of frozen shoulder. In: Itoi E, Arce G, Bain GI, Diercks RL, Guttmann D, et al. Shoulder stiffness. Berlin, Heidelberg: Springer; 2015:21-30

3 Duplay S. De la periarthrite scapulo-humerale. Rev Frat Trav Med. 1896;53:226

4 Codman EA. The shoulder: rupture of the supraspinatus tendon and other lesions in or about the subacromial bursa. Boston: Thomas Todd Co; 1934
5 Zuckerman JD, Rokito A. Frozen shoulder: a consensus definition. J Shoulder Elbow Surg 2011;20(02):322-325

6 Itoi E, Arce G, Bain GI, et al. Shoulder Stiffness: Current Concepts and Concerns. Arthroscopy 2016;32(07):1402-1414

7 Akeson WH, Amiel D, Woo SL. Immobility effects on synovial joints the pathomechanics of joint contracture. Biorheology 1980;17(12):95-110

8 Summers GD, Gorman WP. Bilateral adhesive capsulitis and Hashimoto's thyroiditis. Br J Rheumatol 1989;28(05):451

9 Wang K, Ho V, Hunter-Smith DJ, Beh PS, Smith KM, Weber AB. Risk factors in idiopathic adhesive capsulitis: a case control study. J Shoulder Elbow Surg 2013;22(07):e24-e29

10 Debeer P, Franssens F, Roosen I, Dankaerts W, Claes L. Frozen shoulder and the Big Five personality traits. J Shoulder Elbow Surg 2014;23(02):221-226

11 Cakir M, Samanci N, Balci N, Balci MK. Musculoskeletal manifestations in patients with thyroid disease. Clin Endocrinol (Oxf) 2003;59(02):162-167

12 Reuters VS, Teixeira PdeF, Vigário PS, et al. Functional capacity and muscular abnormalities in subclinical hypothyroidism. Am J Med Sci 2009;338(04):259-263

13 Huang SW, Lin JW, Wang WT, Wu CW, Liou TH, Lin HW. Hyperthyroidism is a risk factor for developing adhesive capsulitis of the shoulder: a nationwide longitudinal population-based study. Sci Rep 2014;4:4183

14 Vanderpump MP. The epidemiology of thyroid disease. Br Med Bull 2011;99:39-51

15 Brenta G, Vaisman M, Sgarbi JA, et al. Task Force on Hypothyroidism of the Latin American Thyroid Society (LATS). Clinical practice guidelines for the management of hypothyroidism. Arq Bras Endocrinol Metabol 2013;57(04):265-291

16 Canaris GJ, Manowitz NR, Mayor G, Ridgway EC. The Colorado thyroid disease prevalence study. Arch Intern Med 2000;160(04): 526-534

17 Schiefer M, Teixeira PFS, Fontenelle C, et al. Prevalence of hypothyroidism in patients with frozen shoulder. J Shoulder Elbow Surg 2017;26(01):49-55

18 Li W, Lu N, Xu H, Wang H, Huang J. Case control study of risk factors for frozen shoulder in China. Int J Rheum Dis 2015;18(05):508-513

19 Milgrom C, Novack V, Weil Y, Jaber S, Radeva-Petrova DR, Finestone A. Risk factors for idiopathic frozen shoulder. Isr Med Assoc J 2008;10(05):361-364

20 Reeves B. The natural history of the frozen shoulder syndrome. Scand J Rheumatol 1975;4(04):193-196

21 Arkkila PE, Kantola IM, ViikariJS, Rönnemaa T. Shoulder capsulitis in type I and II diabetic patients: association with diabetic complications and related diseases. Ann Rheum Dis 1996;55(12):907-914

22 Balci N, Balci MK, Tüzüner S. Shoulder adhesive capsulitis and shoulder range of motion in type II diabetes mellitus: association with diabetic complications. J Diabetes Complications 1999;13 (03):135-140

23 Tighe CB, Oakley WS Jr. The prevalence of a diabetic condition and adhesive capsulitis of the shoulder. South Med J 2008;101(06): 591-595

24 Ramchurn N, Mashamba C, Leitch E, et al. Upper limb musculoskeletal abnormalities and poor metabolic control in diabetes. Eur J Intern Med 2009;20(07):718-721

25 Yian EH, Contreras R, Sodl JF. Effects of glycemic control on prevalence of diabetic frozen shoulder. J Bone Joint Surg Am 2012;94(10):919-923

26 Zreik NH, Malik RA, Charalambous CP. Adhesive capsulitis of the shoulder and diabetes: a meta-analysisi of prevalence. Muscles Ligaments Tendons J 2016;6(01):26-34

27 Zorzano A, Palacín M, Gumà A. Mechanisms regulating GLUT4 glucose transporter expression and glucose transport in skeletal muscle. Acta Physiol Scand 2005;183(01):43-58 